\title{
EMPULSE: a compact terawatt chirped pulse amplification laser for generating coherent $\mathrm{x}$-rays
}

\section{Yousuf Hemani, Marco Galimberti, Davide Bleiner}

Yousuf Hemani, Marco Galimberti, Davide Bleiner, "EMPULSE: a compact terawatt chirped pulse amplification laser for generating coherent X-rays," Proc. SPIE 11886, International Conference on X-Ray Lasers 2020, 1188607 (8 July 2021); doi: 10.1117/12.2595406 


\title{
EMPULSE: A Compact Terawatt Chirped Pulse Amplification Laser for Generating Coherent X-rays
}

\author{
Yousuf Hemani*a,c ${ }^{\text {, }}$ Marco Galimberti ${ }^{\mathrm{b}}$, Davide Bleiner ${ }^{\mathrm{a}, \mathrm{c}}$ \\ ${ }^{a}$ Swiss Federal Institute of Material Sciences (EMPA), 129 Überlandstrasse, 8600, Dübendorf, \\ Zürich, Switzerland; ' Central Laser Facility, STFC, UKRI, Rutherford Appleton Laboratory, Didcot, \\ UK; 'University of Zürich, 190 Winterthurerstrasse, 8057, Zurich, Switzerland
}

\begin{abstract}
Design and development of a compact chirped pulse amplification (CPA) laser is presented. This system will be used to generate coherent tabletop X-rays based on laser-produced plasmas (LPP) for round the clock advanced spectroscopy. The building blocks of the laser are shown and the results from the front-end are laid out. The progress on the amplification stages is presented with a scheme to extract compressed $15 \mathrm{~J}$ laser pulses at the output which are to be characterized and hit on rotating target for generating a plasma with enough population inversion (Ne-like or Ni-Like) as such to enable the emission of Soft X-rays. (C) 2021 The Author(s)
\end{abstract}

Keywords: Plasma, X-ray, Laser, Spectroscopy, Tabletop, Ultrafast, Ne-like, CPA

\section{INTRODUCTION}

The recent decades have seen a rise in high power ultra-intense laser systems in both labs and industries, mainly because of many different applications of these large bandwidth, short duration lasers. These include fundamental research areas such as intense light matter interactions, high energy density research ${ }^{1}$, plasma studies and generation of coherent hard and soft X-rays ${ }^{2}$. These high-power high-energy lasers are commonly enabled by a technique known as chirped pulse amplification (CPA), introduced by Mourou and Strickland, which enables safe energy amplification from solid state gain media using short pulses without experiencing non-linear effects ${ }^{3}$. CPA combined with broadband materials such as $\mathrm{Nd}$ :Glass or Ti:Sapphire, can enable small scale terawatt lasers in a tabletop setting. More research is being done to scale up the power by producing high energy, high repetition rate laser pulses in the lab. A number of such systems exist around the world $d^{4-6}$. One of the applications for such a system is for generating X-rays on a tabletop, which enables all sorts of applications in the domain of X-ray spectroscopy in a laboratory setting.

\subsection{Coherent X-ray Sources}

For experiments, involving spectroscopy, the go-to source for X-rays are synchrotrons, and the advent of XFEL's have opened up promising opportunities in the field of X-ray spectroscopy. These X-ray sources are very attractive for the user because of their high brightness, tunable wavelengths and high repetition rates but these come with a tradeoff as very few of them exist around the world. With a footprint of several kilometers and high cost to build and maintain, it can sometimes hinder basic and continuous research because of the need to get a proposal accepted. That is why; experimentalists have always been keen to develop tabletop X-ray sources, which can mimic to some extent the source characteristics of a beamline. Since the discovery of X-rays, many tabletop sources have been developed, some based on capillary discharge ${ }^{7}$ supporting a single wavelength, others discretely tunable using laser produced plasmas ${ }^{8}$ where a high intensity laser ionizes a solid or a gas target enough to create population inversion for X-ray lasing. Depending on the target and the pump pulse, high brightness X-ray laser lines at several different wavelengths have been characterized ${ }^{9}$. On the other hand, to obtain continuously tunable X-rays on a tabletop, laser wakefield acceleration is the dominant technique, which is in simpler terms a miniaturized synchrotron and finally in order to produce ultrafast X-rays, high harmonic generation (HHG) is the way to go $^{10}$. For spectroscopy experiments, brightness of the X-rays is a key feature as what spectroscopy is in general terms is shining light on processes and tracking them. Figure 1 shows a brightness comparison of tabletop techniques as compared to synchrotrons and XFEL's. In this paper, we discuss the progress on the development of a Terawatt laser, which will enable the emission of soft X-rays based on the laser produced plasma technique in our lab.

*yousuf.hemani@empa.ch; phone+41-58-765-4625; empa.ch

International Conference on X-Ray Lasers 2020, edited by Davide Bleiner,

Proc. of SPIE Vol. 11886, 1188607 - ( ) 2021 SPIE - CCC code:

$0277-786 \mathrm{X} / 21 / \$ 21 \cdot$ doi: $10.1117 / 12.2595406$

Proc. of SPIE Vol. 11886 1188607-1 


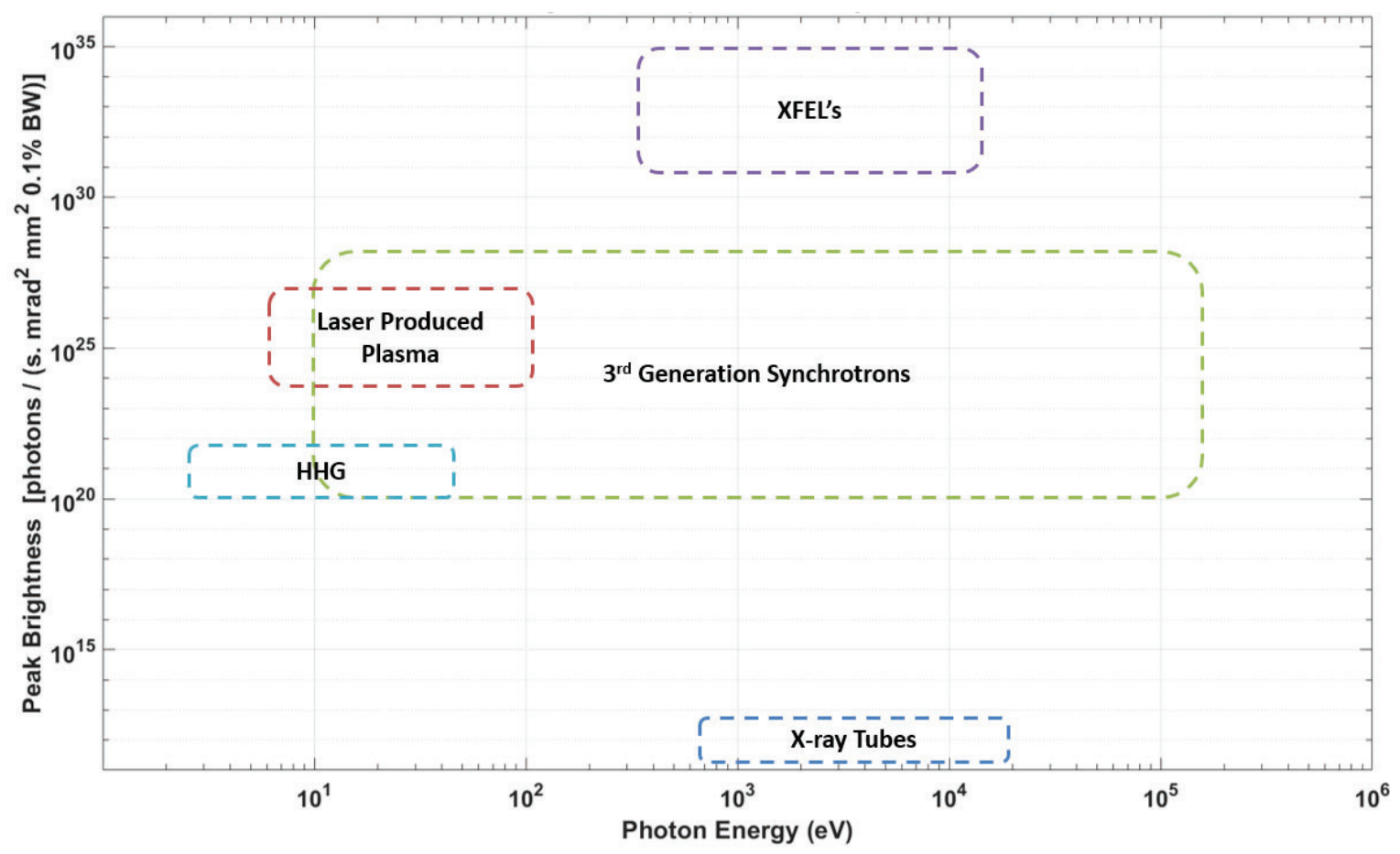

Figure 1. Brightness comparison between sources of X-rays generated in laboratory and at accelerators.

\subsection{Laser Produced Plasma (LPP) X-ray Laser}

Soft X-ray lasers are quasi-monochromatic $\left(\frac{\Delta \lambda}{\lambda} \sim 10^{-5}\right)$, coherent source of photons in the spectral range of $1 \mathrm{~nm}$ to $50 \mathrm{~nm}^{11}$. Soft X-rays based on laser produced plasma are characterized by high photon number ${ }^{12}$, and high spatial coherence ${ }^{13}$ and ultra-narrow linewidth ${ }^{14}$, which make them ideal for applications such as material science ${ }^{15}$, plasma diagnostics ${ }^{16}$, X-ray spectroscopy ${ }^{17}$, lithography ${ }^{18}$, microscopy ${ }^{19}$, etc. The technique to generate soft X-rays in a plasma is to generate a very hot, dense, stable plasma with enough ions and free electrons that it creates a Neon-like or Nickel-like stage ${ }^{20}$. As a principle for any kind of lasing, there needs to be population inversion. In an equilibrium plasma, this is achieved by transient collisional excitation (TCE) ${ }^{21}$. TCE is a popular scheme where a pre-pulse creates a pre-plasma through which a $\mathrm{Ne}$-like or a Ni-like ground stage is established and then the main laser pulse is used to heat up the pre-plasma to create population inversion. The free electrons collide with the ground level electrons and excite them to upper levels where they go back down and lase at a particular wavelength. Figure 2 shows the energy diagram depicting X-ray lasing in a stable Neon-like or Nickel-like ground. To create such a plasma, a short high intensity laser pulse is needed to hit on a gas or a solid target. A combination of transient collisional excitation (TCE) scheme and chirped pulse amplification (CPA) can enable soft X-ray lasing with a pump energy of approximately $10 \mathrm{~J}^{22}$. Another scheme known as Grazing incidence pumping (GRIP), takes the concept of TCE one-step further where the main pulse is shot oblique to the target such as it travels along the plasma column, which further brings down the pump requirements to approximately $1 \mathrm{~J}$ and enables high repetition rates $^{23}$. Figure 3 shows the concept of a line plasma created on a solid rotating target by a high-energy laser pulse for emission of X-rays. A line focus on the target is generated by using cylindrical lenses or in some cases using a spherical or parabolic mirror ${ }^{24}$. Commonly, a double pumping technique is utilized where a long nanosecond pulse of high-energy creates a pre-plasma followed by a short picosecond pulse of high energy to create population inversion. With plasma as a gain medium in a single pass geometry, the phenomenon of amplified spontaneous emission (ASE) occurs through which, soft X-ray laser lines can be generated and characterized using a spectrometer. It has already been shown that large gain length products are possible and because of short gain duration; short intense X-ray pulses can be extracted ${ }^{25}$. A solid medium works better to provide a high gain, high electron density, and Ne-like and Ni-like ground provide better stability as they have one ground state. In a Ne-like scheme, the upper laser states $3 p$ are populated by electron collision from the 
lower $2 \mathrm{p}$ state. Due to rapid decay of $3 \mathrm{~s}$ states to the ground state, population inversion is continued. Ne-like schemes suffers from needing large pump powers while on the other hand almost similar Ni-like scheme is useful for producing lasing at much shorter wavelengths and requires less pumping energy ${ }^{26}$. Ni-like X-ray laser lines have been extracted at many different facilities around the world ${ }^{27-29}$.
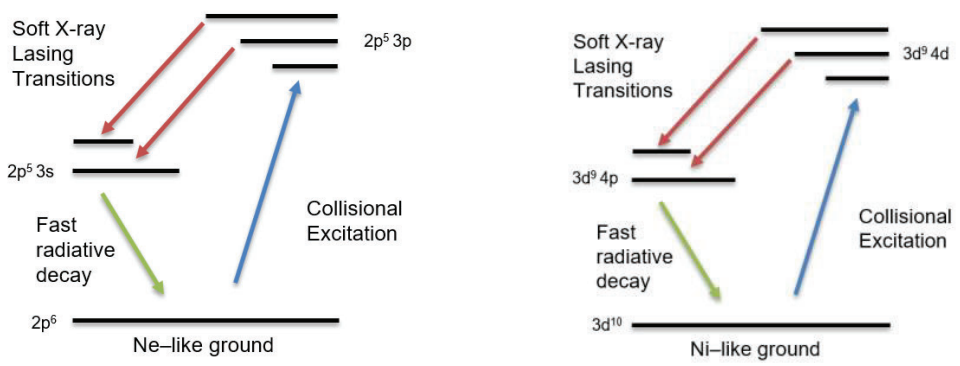

Figure 2. Lasing in a Ne-like or Ni-like ground.

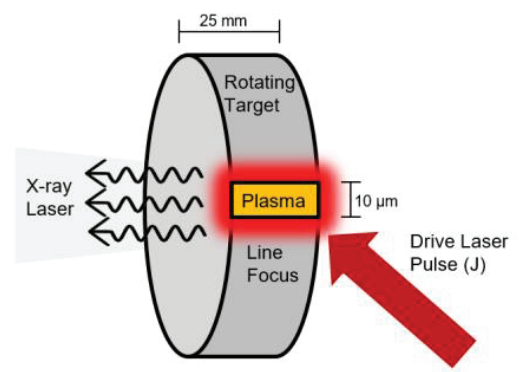

Figure 3. Line focus plasma on a solid target.

\section{EXPERIMENT}

Our tabletop soft x-ray laser system (EMPULSE) consists of a total footprint of three ( $2 \mathrm{~m} \times 1.5 \mathrm{~m})$ optical tables in the lab. The backbone of the system is a femtosecond fiber oscillator laser. In order to get a high peak power, high-energy pulse to create a plasma, we utilize the well-known technique of chirped pulse amplification ${ }^{30}$, where a low energy, high peak power pulse is stretched in time. This enables safe energy amplification of the pulse without optical component damage. The amplified pulse is later compressed to obtain the desired high peak power high-energy output. Figure 4 depicts the steps of CPA. The front-end of EMPULSE consists of a self-developed pulse picker, a 4-pass single grating stretcher and a self-developed regenerative amplifier. To boost the energy of the laser pulse once it is stretched, there are four powerful (Neodymium) Nd glass flash lamp pumped amplifiers and spatial filters. In the end, high energy laser pulses are compressed using a grating pair and delivered into the X-ray lasing chamber on the solid rotating target to create a plasma for X-ray lasing. Figure 5 shows a schematic diagram of the laser system.

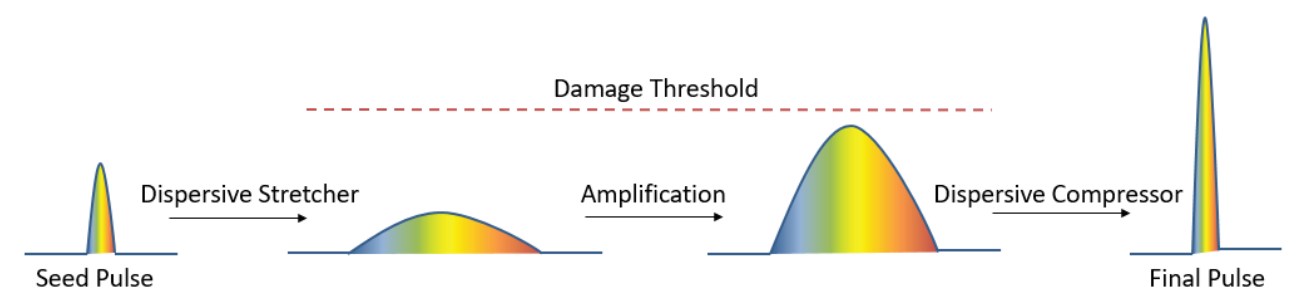

Figure 4. The steps involved in Chirped Pulse Amplification. 


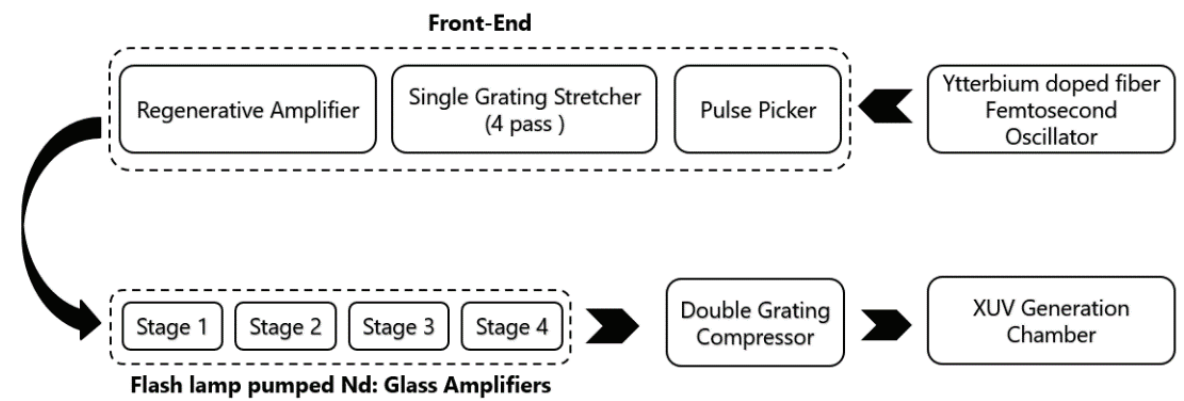

Figure 5. Schematic of building blocks of the system (EMPULSE). Further information on Figure 7.

\subsection{Front End}

The heart of our laser is a commercial Ytterbium doped fiber oscillator, which provides ultrashort pulses with a pulse duration of 120 femtosecond (fs), power of $5 \mathrm{~W}$, repetition rate of $80 \mathrm{MHz}$ and $6 \mathrm{~nJ}$ of energy per pulse. After defining the desired repetition rate, the pulses are picked by a homemade pulse picker consisting of two sets of crossed polarizers and a Pockel cell in between acting as a switch. The pulse is picked up whenever the Pockel cell is triggered. Figure 6 shows a pulse being picked up from the pulse train when triggered by a signal. The picked pulse or pulses are sent to a variable beam expander before they hit the grating. To stretch the pulses in time, a single grating stretcher is implemented with a folded mirror scheme, to utilize the grating in 4-pass stretching geometry, as shown in figure 7. The pulse experiences a strong broadening effect as the grating induces dispersion, affecting the spectral phase. The diffraction grating has 1740 lines $/ \mathrm{mm}$. The pulses are stretched to approximately one nanosecond (ns) duration, which can be measured by using a fast diode or the stretching factor can also be determined mathematically by using the grating equation and the final pulse duration can be estimated depending on distance between the gratings and angle of incidence of the laser pulse $\mathrm{e}^{31}$. One of the limiting factors of the stretcher is the bandwidth of the grating, resulting in a spectra with less wavelengths, when the pulse exits the stretcher, as shown in Figure 8.

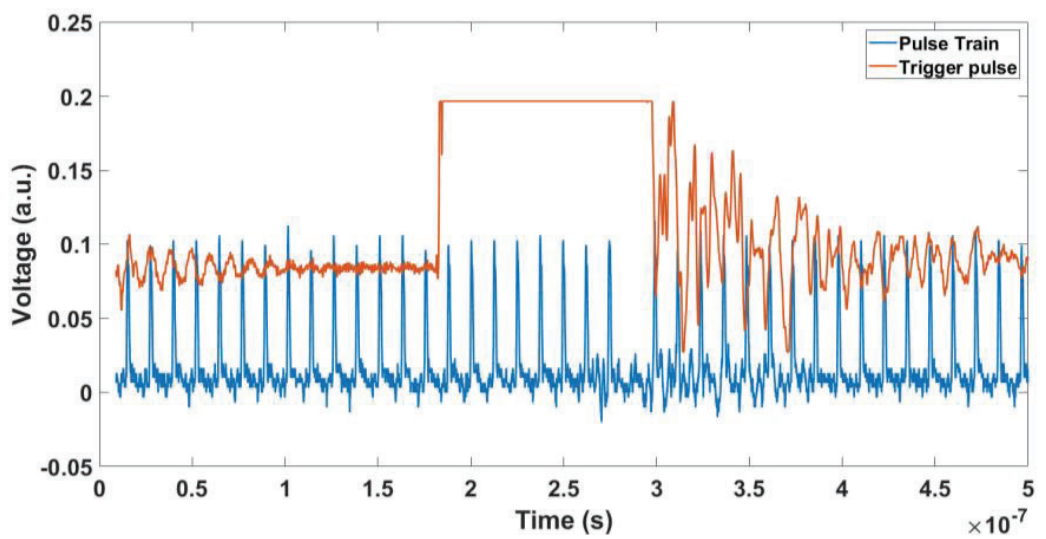

Figure 6. The trigger pulse for the Pockel cell (red) and the pulse train coming from the oscillator (blue) are shown. The missing pulse from the pulse train is the picked pulse, which is sent to the stretcher. 


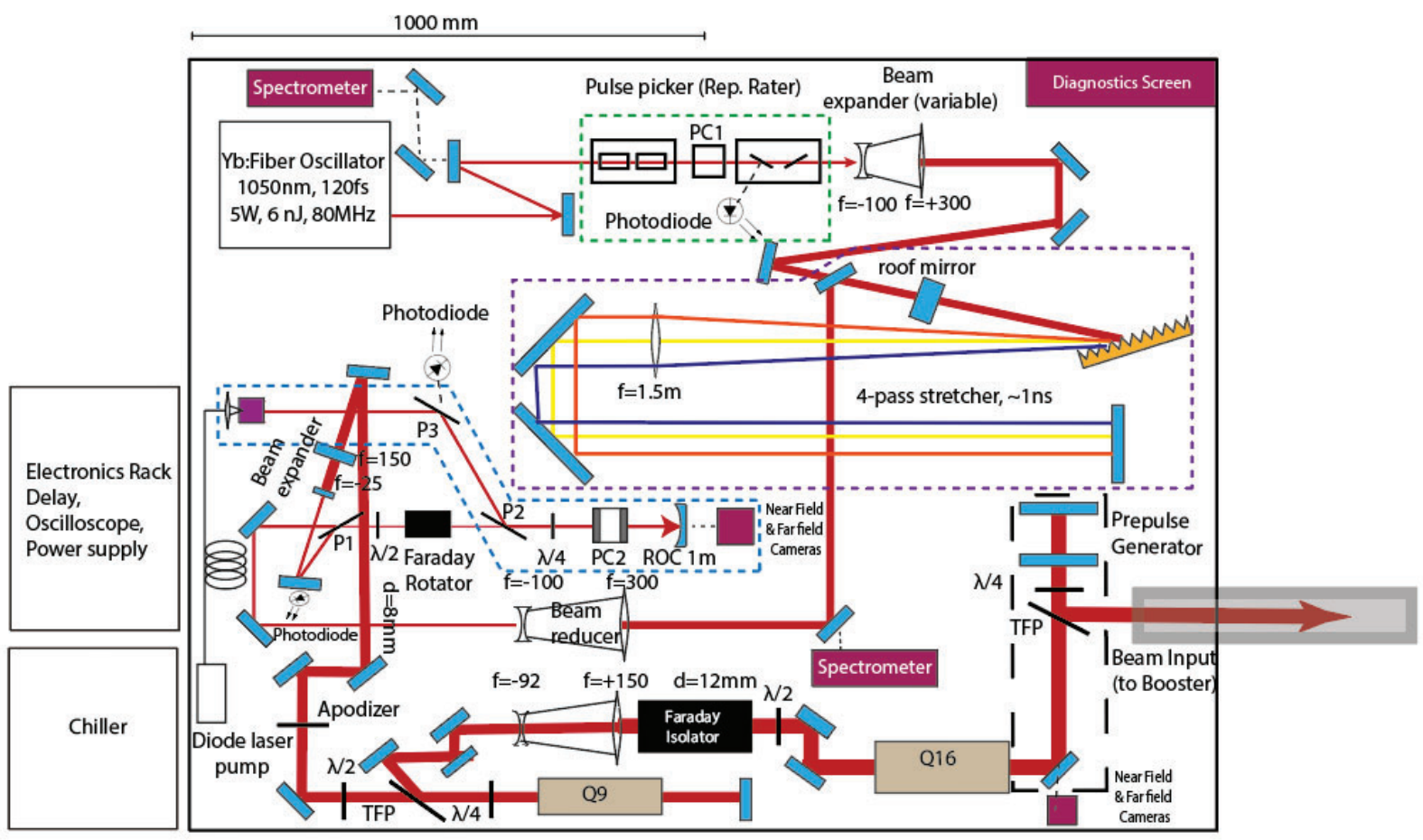

Figure 7. Optical Design Sketch for the Front-end.

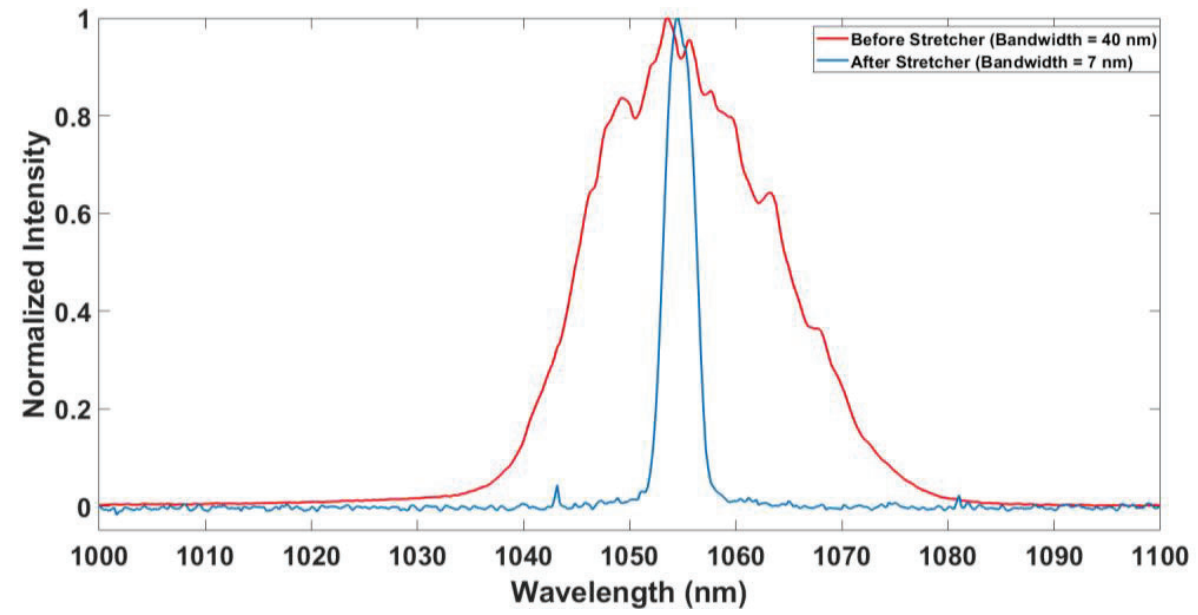

Figure 8. Spectra for the oscillator (red) shows much broader bandwidth, while spectra after stretcher (blue) shows a narrower bandwidth range.

For characterization of the pulse output from the stretcher, a wave front sensor and a few CCD cameras are used to measure the astigmatism and beam profile in near field and far field propagation window. Figure 9 shows the near field profile (left) and far field beam profile (right). The beam looks perfectly Gaussian in the near field although there is astigmatism seen in the far field propagation, which can be corrected by using an aperture for instance. 

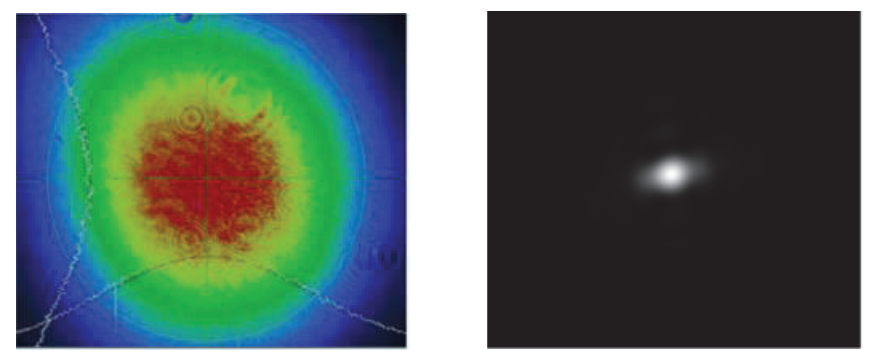

Figure 9. Near field beam profile (left) and far field beam profile (right) for the output of laser pulse from the stretcher.

After the stretcher, the beam size is reduced, before the pulse enters the regenerative amplifier cavity for pre-amplification. A regenerative amplifier resembles a laser cavity with a gain medium and a pumping mechanism. A seed pulse enters the cavity; it is amplified in several round trips and exits the cavity at the desired energy controlled by the timing delay. The regenerative amplifier implemented for this setup consists of a double channel Pockels Cell, a faraday rotator, a quarter wave plate, a half wave plate, three sets of polarizers and a curved end mirror, as shown in figure 7. It is pumped by Quasi $\mathrm{CW}$ laser diode with a central wavelength of $808 \mathrm{~nm}$ at a power of $70 \mathrm{~W}$. The gain medium is Neodymium Phosphate glass (LHG $8 \mathrm{p} / \mathrm{p} \mathrm{1 \%} \mathrm{Nd).} \mathrm{Both} \mathrm{the} \mathrm{gain} \mathrm{medium} \mathrm{and} \mathrm{the} \mathrm{diode} \mathrm{are} \mathrm{connected} \mathrm{to} \mathrm{a} \mathrm{chiller} \mathrm{kept} \mathrm{at} 19 \mathrm{C}$ to eliminate temperature effects. The length of the regen cavity is 1 meter, while the time for one roundtrip is $6.6 \mathrm{~ns}$. The total number of round trips that the pulse makes before exiting the cavity is approximately 40. For monitoring the output of the regenerative amplifier, two CCD cameras are installed at the back of the end mirror to monitor the beam profile in near field and far field propagation on the optical table. A silicon diode detector attached to an oscilloscope monitors the output ramp-up signal, the energy is measured using a power sensor, and an energy output reader. The results from the regenerative amplifier are shown in the next section.

\subsection{Amplification Chain}

As shown in figure 7, for main pulse amplification, powerful Nd glass flash lamp pumped amplifiers are used. Flash lamp pumped glass rod amplifiers provide large gain and increase the energy of pulse drastically. In our setup, four amplifier modules are implemented with different gain media lengths. On the first table there is Q9 and Q16 having rods 9 mm and $16 \mathrm{~mm}$ respectively. To take advantage of the full face of the gain media, the pulse output from the regenerative amplifier is expanded first to 8mm for Q9 and then 12mm for Q16. Q9 is operated in double pass mode while Q16 is operated in single pass mode. The amplifiers are connected to a flash lamp control module and internal chiller for regulation.

The whole amplification procedure is governed by a timing synchronization scheme. Expected output of energy per pulse after Q9 is around 2mJ in single pass and $20 \mathrm{~mJ}$ in double pass. Q16 will further amplify the pulse up to $300 \mathrm{~mJ}$. A faraday isolator is used to prevent any back reflections in the system. A further amplification scheme is planned to get a $15 \mathrm{~J}$ energy per pulse at the end using two more amplifier heads. This stage is under development and out of the scope for this paper. Figure 10 shows the planned amplification scheme for EMPULSE.

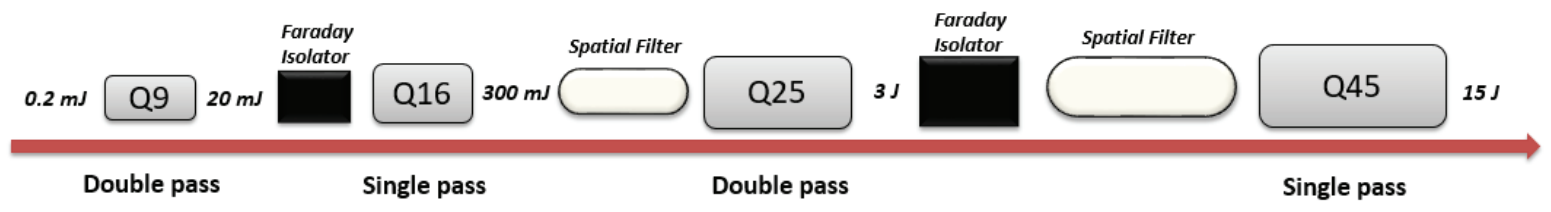

Figure 10. Planned amplification scheme for EMPULSE 


\section{RESULTS}

\subsection{Regenerative Amplifier}

The results from the regenerative amplifier are shown in this section. The pulse repetition rate is $2 \mathrm{~Hz}$ after the picker. The pulse is stretched up to one nanosecond after the grating and then sent into the regenerative amplifier for pre-amplification stage. The output of the regen is up to $0.2 \mathrm{~mJ}$ on average. The near field profile is more flat-top than Gaussian as shown in figure 11. Figure 12 shows the ramp-up operation of the amplifier showing the increase in energy with the number of roundtrips. The final energy extracted from the regen is shown in figure 13, which is measured over a period of 1 hour. The root mean square value is $0.19 \mathrm{~mJ}$. The standard deviation of the data is $0.0068 \mathrm{~mJ}$, with a variability range of 0.196 $\mathrm{mJ}$ to $0.182 \mathrm{~mJ}$, while relative standard deviation is found to be $3.6 \%$. The maximum fluctuation in the data is at $0.22 \mathrm{~mJ}$ and minimum at $0.17 \mathrm{~mJ}$, therefore the absolute difference in peak fluctuations comes out to be $0.048 \mathrm{~mJ}$.

Figure 11. Beam profile at the output of the regenerative amplifier.

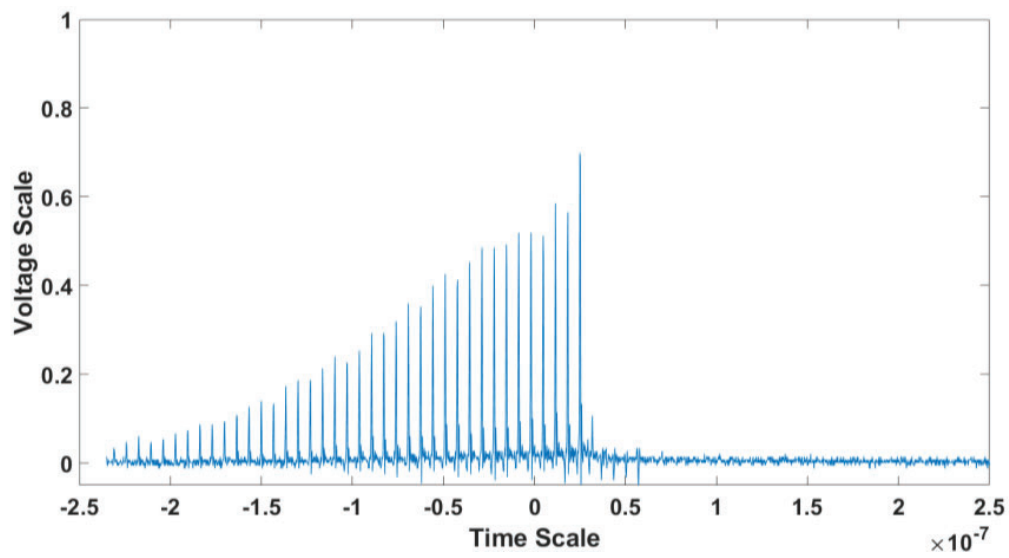

Figure 12. The ramp-up operation of the regenerative amplifier with increasing signal every roundtrip.

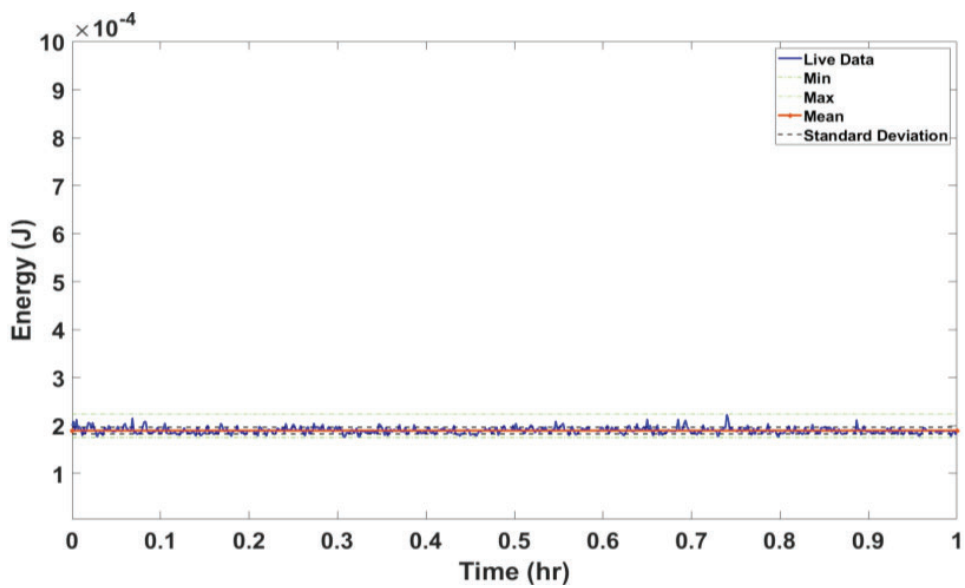

Figure 13. The energy output of the regenerative amplifier over a period of 1 hour at $2 \mathrm{~Hz}$ 


\section{DISCUSSION}

A pre-pulse generator will be implemented to take advantage of the double pumping phenomenon. Also, B-integral has to be investigated to avoid dangerous non-linear effects in the amplification chain. The second table will consist of the rest of the two amplification head Q25 with $25 \mathrm{~mm}$ rod and Q45 with $45 \mathrm{~mm}$ rod. Vaccum spatial filters will be used to clean the beam and image it on the face of the respective rods and to deliver the beam to the compressor. The compressor will be implemented in a two grating double pass geometry to compress the pulse down to approximately few picoseconds. A commercial single shot auto correlator can be used to characterize the beam before sending it inside the X-ray generation chamber under high vacuum. An optical system has to be designed to precisely create a line focus onto a solid rotating target. We expect to have one shot per minute for the laser pulse hitting on the target. The X-rays can be collected and characterized from the output of the X-ray chamber.

\section{CONCLUSION}

The initial development phase of a tabletop X-ray source based on laser-produced plasma (LPP) is demonstrated. The specifications and outcomes of the front end are shown to be comparable to contending systems (see also Sect. 1-4 [32]). The high-energy amplification scheme is put forward and a plan for the development of the next phase is proposed. The last phase will consist of pulse compression, plasma generation and collection of X-rays. Soft X-rays laser lines from different targets will be characterized and used for different spectroscopy experiments; which is indeed the end goal. Coherent, short, bright X-rays can be used for many different applications such as X-ray microscopy, Xray spectroscopy and X-ray lithography in a home lab setting.

\section{REFERENCES}

[1] Kumar, G. R., "High energy density science with tabletop terawatt lasers," J. Phys.: Conf. Ser. 244(1), 012008 (2010)

[2] Martellucci, S., Bellecci, C., Francucci, M., Gaudio, P., Richetta, M., Toscano, D., Rydzy, A., Gelfusa, M. and Ciuffa, P., "Soft X-ray generation by a tabletop Nd:YAG/glass laser system," J. Phys.: Condens. Matter 18(33), S2039-S2044 (2006).

[3] Perry, M. D. and Mourou, G., "Terawatt to Petawatt Subpicosecond Lasers," Science 264(5161), 917-924 (1994)

[4] Danson, C. N., Angood, S., Barzanti, L. J., Bradwell, N., Collier, J. L., Damerell, A. R., Edwards, C. B., Johnson, C., Key, M. H., Neely, D., Nightingale, M., Norreys, P. A., Pepler, D. A., Rodkiss, D. A., Ross, I. N., Ryves, P., Thompson, N., Trentelman, M., Walsh, F. N., et al., "Design and characterization of the VULCAN Nd:glass laser to give focused intensities of greater than $10^{19 \mathrm{~W} / \mathrm{cm} 2}$," Solid State Lasers for Application to Inertial Confinement Fusion: Second Annual International Conference, M. L. Andre, Ed., SPIE (1997).

[5] Badziak, J., Chizhov, S. A., Kozlov, A. A., Makowski, J., Paduch, M., Tomaszewski, K., Vankov, A. B. and Yashin, V. E., "Picosecond, terawatt, all-Nd:glass CPA laser system," Optics Communications 134(1-6), 495502 (1997).

[6] Bleiner, D., "Coherent Short-Wavelength Plasma Radiation for Lab-scale Nano-inspection Tools," [Springer Proceedings in Physics], Springer International Publishing, 39-44 (2014).

[7] Macchietto, C. D., Benware, B. R. and Rocca, J. J., "Generation of millijoule-level soft-x-ray laser pulses at a 4$\mathrm{Hz}$ repetition rate in a highly saturated tabletop capillary discharge amplifier," Opt. Lett. 24(16), 1115 (1999)

[8] Albrecht, M., Kozlova, M. and Nejdl, J., "Radiation properties of Ni-like molybdenum X-ray laser at PALS," Xray Lasers and Coherent X-ray Sources: Development and Applications, A. Klisnick and C. S. Menoni, Eds., SPIE (2017). 
[9] Daido, H., "Review of soft x-ray laser researches and developments," Rep. Prog. Phys. 65(10), 1513-1576 (2002).

[10] Bleiner, D., "Tabletop Beams for Short Wavelength Spectrochemistry," Spectrochimica Acta Part B: Atomic Spectroscopy, 105978 (2020).

[11] Suckewer, S. and Jaeglé, P., “X-Ray laser: past, present, and future,” Laser Phys. Lett. 6(6), 411-436 (2009).

[12] Rus, B., Mocek, T., Kozlová, M., Polan, J., Homer, P., Stupka, M., Tallents, G. J., Edwards, M. H., Mistry, P., Whittaker, D. S., Booth, N., Zhai, Z., Pert, G. J., Dunn, J., Nelson, A. J., Foord, M. E., Shepherd, R., Rozmus, W., Baldis, H. A., et al., "Development and applications of multimillijoule softX-ray lasers," Journal of Modern Optics 54(16-17), 2571-2583 (2007)

[13] Nishikino, M., Tanaka, M., Nagashima, K., Kishimoto, M., Kado, M., Kawachi, T., Sukegawa, K., Ochi, Y., Hasegawa, N. and Kato, Y., "Demonstration of a soft-X-ray laser at $13.9 \mathrm{~nm}$ with full spatial coherence," Phys. Rev. A 68(6) (2003)

[14] Bleiner, D., "Comparison of laboratory-scale XUV laser with xFELs," Short-Wavelength Imaging and Spectroscopy Sources, D. Bleiner, Ed., SPIE (2012).

[15] Mocek, T., Rus, B., Kozlová, M., Polan, J., Homer, P., Juha, L., Hájková, V. and Chalupský, J., "Single-shot soft $\mathrm{X}$-ray laser-induced ablative microstructuring of organic polymer with demagnifying projection," Opt. Lett. 33(10), 1087 (2008).

[16] Da Silva, L. B., Barbee, T. W., Jr., Cauble, R., Celliers, P., Harder, J., Lee, H. R., London, R. A., Matthews, D. L., Mrowka, S., Moreno, J. C., Ress, D., Trebes, J. E., Wan, A. and Weber, F., "X-ray lasers for high density plasma diagnostics (invited)," Review of Scientific Instruments 66(1), 574-578 (1995).

[17] Mantouvalou, I., Witte, K., Martyanov, W., Jonas, A., Grötzsch, D., Streeck, C., Löchel, H., Rudolph, I., Erko, A., Stiel, H. and Kanngießer, B., "Single shot near edge x-ray absorption fine structure spectroscopy in the laboratory,” Appl. Phys. Lett. 108(20), 201106 (2016).

[18] Marconi, M. C., Wachulak, P. W., Urbanski, L., Isoyan, A., Jiang, F., Cheng, Y. C., Rocca, J. J., Menoni, C. S. and Cerrina, F., "Tabletop soft X-ray lithography," Soft X-Ray Lasers and Applications VIII, J. Dunn and G. J. Tallents, Eds., SPIE (2009).

[19] Bleiner, D., Staub, F. and Balmer, J. E., "EUV microscopy using a lab-scale x-ray laser source,” X-Ray Lasers and Coherent X-Ray Sources: Development and Applications IX, J. Dunn and A. Klisnick, Eds., SPIE (2011).

[20] Bleiner, D., Arbelo-Pena, Y., Masoudnia, L. and Ruiz-Lopez, M., "Table-top x-ray lasers using a plasma gainmedium: limits and potentials," Phys. Scr. T162, 014050 (2014).

[21] Afanas'ev, Y. V. and Shlyaptsev, V. N., "Formation of a population inversion of transitions in Ne-like ions in steady-state and transient plasmas,” Sov. J. Quantum Electron. 19(12), 1606-1612 (1989).

[22] Shlyaptsev, V. N., Nickles, P. V., Schlegel, T., Kalachnikov, M. P. and Osterheld, A. L., "Tabletop x-ray laser pumped with subnanosecond and picosecond pulses," Ultrashort Wavelength Lasers II, S. Suckewer, Ed., SPIE (1994)

[23] Keenan, R., Dunn, J., Shlyaptsev, V. N., Smith, R. F., Patel, P. K. and Price, D. F., "Efficient pumping schemes for high average brightness collisional x-ray lasers," Soft X-Ray Lasers and Applications V, E. E. Fill and S. Suckewer, Eds., SPIE (2003).

[24] Bleiner, D., Balmer, J. E. and Staub, F., "Line focusing for soft x-ray laser-plasma lasing,” Appl. Opt. 50(36), 6689 (2011). 
[25] Jamelot, G., Carillon, A., Dhez, P., Gauthe, B., Jaegle, P., Klisnick, A. and Raucourt, J. P., "X-ray laser using lithium-like recombination scheme: effects of plasma inhomogeneity," Ultrashort Wavelength Lasers, S. Suckewer, Ed., SPIE (1992).

[26] Svelto, O., Longhi, S., Della Valle, G., Huber, G., Kück, S., Pollnau, M., Hillmer, H., Kusserow, T., Engelbrecht, R., Rohlfing, F., Kaiser, J., Malz, R., Marowsky, G., Mann, K., Simon, P., Rhodes, C. K., Duarte, F. J., Borsutzky, A., L'huillier, J. A., et al., "Lasers and Coherent Light Sources," [Shamdheorodfaserand Optics], Springer Berlin Heidelberg, 641-1046 (2012).

[27] Guilbaud, O., Edwards, M., Klisnick, A., Ros, D., Jamelot, G., Joyeux, D., Phalippou, D., Tang, H., Neumayer, P., Ursescu, D., Tallents, G. J., Kuehl, T., Cassou, K., Bouhouch, K., Kado, M., Nishikino, M., Sukegawa, K., Kishimoto, M., Ishino, M., et al., "Near-field imaging of Ni-like silver transient collisional x-ray laser,” Soft X-Ray Lasers and Applications V, E. E. Fill and S. Suckewer, Eds., SPIE (2003).

[28] Lewis, C. L. S. and Tallents, G. J., "A review of Ni-like X-ray laser experiments undertaken using the VULCAN laser at the Rutherford Appleton Laboratory," Comptes Rendus de l'Académie des Sciences - Series IV - Physics 1(8), 1053-1063 (2000).

[29] Rocca, J. J., Furch, F. J., Reagan, B. A., Wang, Y., Alessi, D., Martz, D., Luther, B. M., Curtis, A. H., Meehan, S. P., Domingue, S. and Kemp, D., "Recent Advances of Table-Top Soft X-ray Lasers," Lasers, Sources and Related Photonic Devices, OSA (2010).

[30] Strickland, D. and Mourou, G., “Compression of amplified chirped optical pulses," Optics Communications 55(6), 447-449 (1985).

[31] Zhong, Z., Gong, W., Jiang, H., Gu, H., Chen, X. and Liu, S., "Investigation of Spatial Chirp Induced by Misalignments in a Parallel Grating Pair Pulse Stretcher," Applied Sciences 10(5), 1584 (2020).

[32] D. Bleiner, The Science and Technology of X-ray Lasers: A 2020 Update Proc. SPIE 11886, 1188602 (2021) 